\title{
Temporal Variation in Prehistoric Nubian Crania 1
}

\author{
DAVID S. CARLSON \\ Center for Human Growth and Development, University of Michigan, \\ Ann Arbor, Michigan 48109
}

\begin{abstract}
KEY WORDS Craniofacial evolution - Mastication - Cephalometrics $\cdot$ Multiple discriminant analysis $\cdot$ Nubia.
\end{abstract}

\begin{abstract}
Much of the earlier work on the prehistory of Sudanese Nubia has emphasized discontinuity between early Nubian populations. However, recent investigations suggest the converse - that a remarkable degree of cultural and biological continuity exists among indigenous Nubian groups, perhaps as far back as the Paleolithic. Thus, cultural and biological differences between Nubian populations can be most effectively perceived as the result of in situ evolutionary development.

The present analysis has two major purposes: (1) to describe the morphological differences in the craniofacial complex between indigenous Nubian populations extending from the A-Group (c. 3,400 B.C.) through the Christian (c. 1,500 A.D.) horizons; and (2) to account for these differences within an evolutionary framework. The multiple discriminant analysis of radiographically derived variables revealed a trend from a substantially lower and more elongated cranial vault to a shorter and taller vault throughout the almost 5,000 year time span. Associated with this pattern was a tendency for the face to become more inferiorly-posteriorly located with respect to the vault in the latter groups. Finally, the masseter and temporalis muscles underwent a reduction and slight relocation through time. We speculate that this trend may be associated with behavioral changes associated with transition from a hunting and gathering to a totally agricultural subsistence pattern.
\end{abstract}

The extent of archeological investigation of Lower Nubia over the past century has led some authors to conclude that, archeologically, Lower Nubia is one of the best known areas in the world (Adams, '66; Lasker, '72). A considerable amount of the early interest in human skeletal remains from the prehistoric Nile River Valley was brought about by questions relating to the racial composition of early Nilotic peoples. Primary concern here rested on the origin of ancient Egyptian populations, and precipitated investigation of the racial composition of prehistoric Nubians for comparative purposes (Collett, '33; Stoessiger, '27). As a result, anthropometric analysis of Nubian populations, both skeletal and contemporary, has been closely intertwined with archeological investigation of the culture history of the Nile River valley. In- deed, a consistent element in virtually every study of Nubian culture history, especially prior to 1960 , is a concern for the origin and "racial identity" of Nubian populations.

The prevailing opinion during the earlier half of this century among Nubian scholars was that two racial "types" existed in the Nile River valley. Morant ('25, '35) suggested that an earlier "Upper Nile type," which exhibited a high concentration of "Negroid traits," and a later "Lower Nile type", which was largely lacking in "Negroid" features, existed in Nubia. Like several of his contemporaries (Stoessiger, '27; Derry, '09), Morant noted a continuity between earlier Badarian populations, dat-

1 This research was supported in part by Wenner-Gren Grant No. 2959, and United States Public Health Service Grant NIH DE-03610. 
ing from c. 4,000 B.C. in Upper Egypt, and later predynastic populations in both Egypt and Lower Nubia. The decrease in "Negroid traits" from the Badarian to the predynastic populations was interpreted as evidence for admixture between the two basic types, "Negroids" to the south and "Caucasoids" to the north (Morant, '35). This basic theme of racial admixture to explain morphological changes repeats itself throughout the history of Nubian archeological investigation, and remains well represented in the recent literature (e.g. Baumgartel, '65; Burnor and Harris, '65; Crichton, '66; Wiercinski, '67; de Villiers, '68; Strouhal, '71; Rightmire, '74).

Interpretations of the succession of cultural traditions in Lower Nubia were, similarly, based on what Adams ('66) has termed the "multiple migration hypothesis." The suggestion that culture change in Lower Nubia was the result of migrations of alien peoples led to a search for racial differences between the skeletal populations associated with the major cultural traditions during the past 5,000 years as an explicit or implicit explanation of cultural development (e.g. Reisner, '09; Junker, '28; Batrawi, '35; Arkell, '61). “Advances" in Nubian culture tended to be perceived as coincident with an increase in Caucasoid (Egyptian) types. Similarly, cultural "decline" or "degeneration" was explained by an increase in "Negroid element." The racist ideas on which these assumptions are founded are clearly evident.

The theoretical foundation for the use of the racial model to explain culture change in skeletal morphology in Nubia has received widespread criticism only recently (Adams, '66; Van Gerven, Carlson and Armelagos, '73). MacGaffey ('66), for example, noted that anthropologists have helped to maintain and perpetuate the "racial myths" particular to Nubian prehistory by failing to dispel the biological inconsistencies inherent in the typological approach. Similarly, Adams ('66, '67, '68, 70 ) has been extremely critical of the use of the racial model to account for culture change in early Nubia, noting that the archeology of Nubia demonstrates a "continuum of cultural evolution... involving the same basic population from beginning to end" ('66: p. 29).

Several analyses of skeletal remains from Lower Nubia have cast considerable doubt on the multiple-migration view of Nubian prehistory. In a craniometric analysis of Nubian populations Batrawi ("46) concluded that the indigenous populations of Lower Nubia were a single biological population extending from the A-Group (c. 3,400 B.C.) to the Christian horizons (c. 1,500 A.D.). Mukherjee et al. (55) reanalyzed the same material using the Mahalanobis $\mathrm{D}^{2}$ and similarly concluded that all of the indiginous populations of lower Nubia could be considered as a single biologically homogeneous population. This general conclusion was recently re-affirmed by Nielsen (70), who noted that the A-Group and C-Group, in particular, were probably composed of a single homogeneous population. Those craniometric differences which do occur between the A-Group and C-Group are most likely due to evolutionary change rather than to hybridization between biologically distinct peoples. Nielsen further concluded that, despite some differences in the XGroup population, the Meroitic, X-Group, and Christian horizons all form a common biological population.

Recent analyses of discrete skeletal and dental variants in early Nubian populations have led to similar conclusions (Greene, '66, '72; Berry et al., '67; Berry and Berry, '72). In fact, Greene ('72) noted that the appearance of rare cusp variants, such as split maxillary hypocone, in the dentition of populations from the Nubian Mesolithic through the later groups suggests a much longer continuity than previously realized.

In sum, several independent analyses of skeletal remains from early Nubian populations and recent considerations of the archeology of Lower Nubia are in agreement that there is no evidence for any intrusion by alien peoples for well over the last 5,000 years. Skeletal remains from an- 
cient Nubian populations thus offer unique opportunities for research in human biology. The Nile River valley is perhaps the only area where one can obtain vast amounts of biological data from relatively homogeneous human populations, and where "the stages of microevolution and adaptation can be followed accurately in time and space with few outside influences" (Masali, '72: p. 196). While most research using ancient Nubian skeletal remains has followed primarily a historically oriented approach to problems of cultural and biological change, it is clearly appropriate to use ancient Nubian remains in problems of an evolutionary, processual design.

The general purpose of this research is to re-evaluate Nubian craniofacial morphology in light of our current understanding of Nubian prehistory. The more specific goals are two-fold: (1) to describe the biometric relationships exhibited by the populations, and; (2) to account for these relationships on the basis of differences in specific morphological features. Variation between the temporally successive Nubian populations over almost a 5,000 year time period is quantified within a multivariate framework, through the utilization of the technique of multiple discriminant analysis. The differences between populations are discussed via a detailed examination of the extracted discriminant functions. This approach provides a means of describing interpopulation variation and identifying factors accounting for such variation. This analysis, furthermore, provides an understanding of morphometric differences between Nubian populations as time-related phenomena, thus providing a foundation for more complete analyses of temporal variation in the recent human skull.

\section{MATERIALS}

The cranial remains used in this analysis were recovered by the Scandinavian Joint Expedition to Sudanese Nubia (Nielsen, 70). The Scandinavian concession in Lower Nubia extended $60 \mathrm{~km}$ along the east bank of the Nile River, beginning at the Sudanese-Egyptian border to the north (fig. 1). The entire concession was extensively surveyed, and the 73 sites discovered were all completely excavated.

The burials represent the time span between c. 3,400 B.C. and 1,500 A.D., and include all the major Nubian cultural horizons during that time (table 1). All cultural assignments of the burials were made on the basis of grave goods and burial type. A more detailed account of the excavations can be obtained in the reports of the Scandinavian Joint Expedition (Nordström and Haland, '72; Nordström and Ojamaa-Koskinen, '73; Holthoer et al., '73; Säve-Söderbergh, '62, '63, '64' Møllerup and Oldenburg, '73).

Only complete non-senile adult crania with no obvious pathological deformity that might affect cranial shape were included in the analysis. Male and female crania were found in approximately equal proportions for each culture group, and were therefore combined for analysis.

\section{METHODS}

Crania were radiographed in the median sagittal plane with each skull held in place by a Björk cephalostat (Krogman and Sas-

TABLE 1

Sample size and dates of the five Nubian archeological populations

\begin{tabular}{lcccc}
\hline \multirow{2}{*}{ Population } & \multicolumn{3}{c}{ Sex } \\
\cline { 3 - 4 } & $\mathrm{N}$ & Male & Female & Dates \\
\hline Christian (Ch) & 39 & 21 & 18 & $550-1,500$ A.D. \\
X-Group (X) & 67 & 35 & 32 & $350-550$ A.D. \\
Meroitic (M) & 57 & 27 & 30 & $0-350$ A.D. \\
C-Group (C) & 40 & 20 & 20 & 2,400-1,200 B.C. \\
A-Group (A) & 12 & 5 & 7 & $3,400-2,400$ B.C. \\
\hline
\end{tabular}




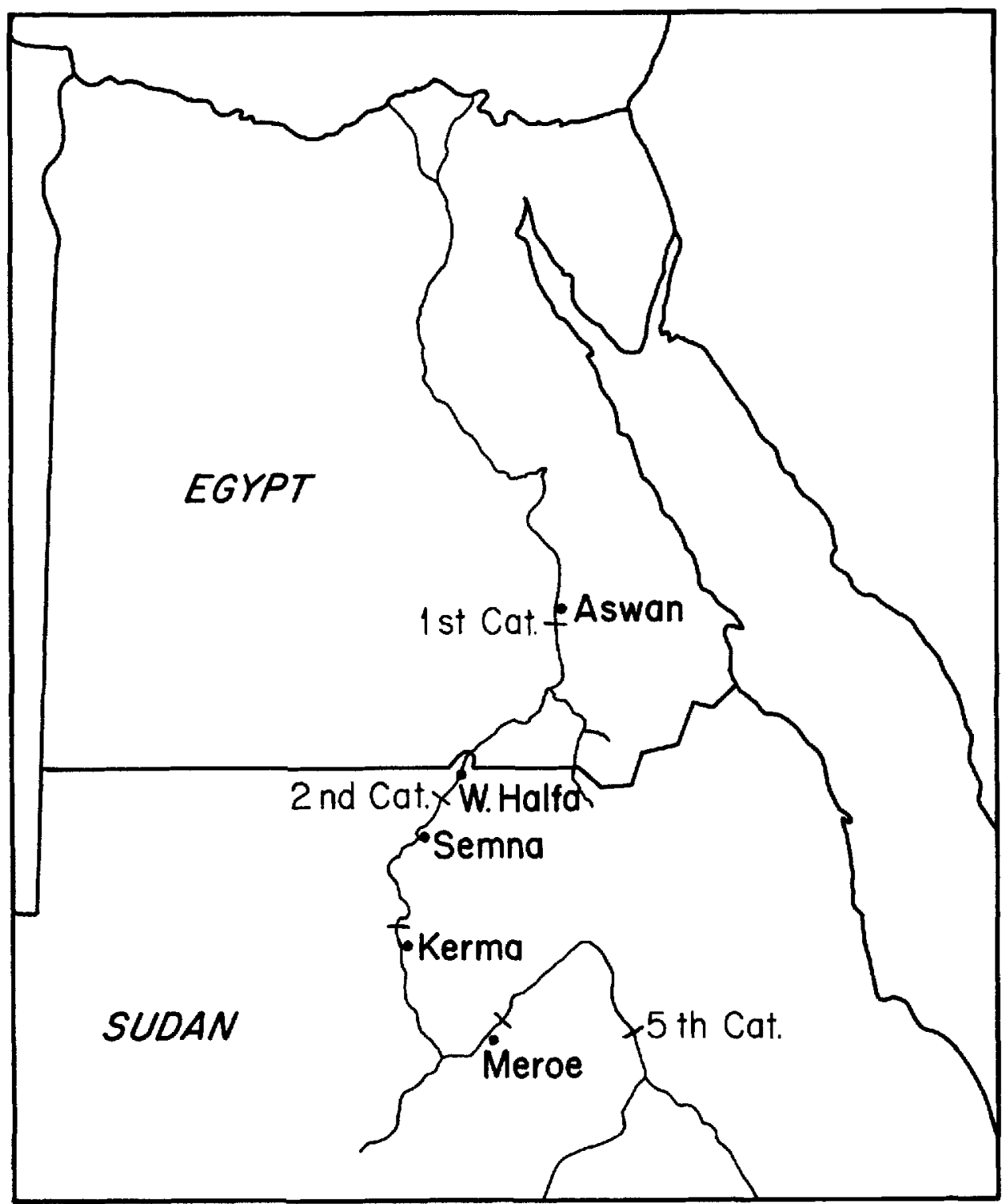

Fig. 1 Map of the Nile River valley through the 6th Cataract. The Scandinavian Joint Expedition concession area extended from the Egyptian-Sudanese border to the north through the 2nd Cataract and the town of Semna to the south.

souni, '57). Distance between the $\mathrm{x}$-ray anode and skull was kept constant at 155 $\mathrm{cm}$, maintaining magnification at or below 5\% (Krogman and Sassouni, '55).

Following the procedures recently developed by Walker for the analysis of roentgenographic cephalograms (Walker, '72; Walker and Kowalski, '73), reference points for each skull were recorded as spatial coordinates, which were then transformed into linear and angular dimensions between designated sets of points for statistical analysis (fig. 2).

\section{Cephalometric variables}

A total of 37 linear and angular measure- 
ments were determined from the radiographic cephalogram of each complete skull.

\section{Cranial base}

Anterior Cranial Base Length: the distance from sella (s) to nasion (n).

Posterior Cranial Base Length: the distance from sella (s) to opisthocranion (op).

Median Cranial Base Length: the distance between sella (s) and basion (ba).

Median Cranial Base Angle: the angle between the nasion-sella line (NSL) and a line connecting basion (ba) and sella (s).

\section{Cranial vault}

Anterior Cranial Fossa Length: the distance from sella (s) to the midfrontal point (mfp).

Median Cranial Fossa Height: the distance from sella (s) to bregma (b).

Posterior Cranial Fossa Length: the distance from sella (s) to lambda (la).

Total Cranial Length: the distance from glabella ( $\mathrm{g}$ ) to opisthocranion (op).

Cranial Height: the distance from basion (ba) to bregma (b).

Frontal Chord: the straightline distance from nasion (n) to bregma (b).

Parietal Chord: the straightline distance from bregma (b) to lambda (la).

Midfrontal Angle: the angle formed at glabella (g) by lines from the midfrontal point (mfp) and nasion (n).

\section{Prognathism}

Maxillary Basal Prognathism: the angle formed by NSL and a line through nasion (n) and subspinale (ss).
Maxillary Alveolar Prognathism: the angle formed by NSL and a line through nasion (n) and prosthion (pr).

Mandibular Basal Prognathism: the angle formed by NSL and a line through nasion (n) and supramentale (sm).

Mandibular Alveolar Prognathism: the angle formed by NSL and a line through nasion (n) and infradentale (id).

\section{Maxillary and mandibular size and relations}

Maxillary Length: the distance from the posterior nasal spine (pns) to subspinale (ss).

Posterior Facial Angle: the angle formed by NSL and a line connecting sella (s) with the posterior nasal spine (pns).

Inclination of Maxilla to NSL: the angle formed by the nasal line and NSL.

Posterior Upper Face Height: the distance from sella (s) to the posterior nasal spine (pns)

Upper Face Length: the distance from basion (ba) to prosthion (pr).

Ramus Height: the distance from articulare (ar) to gonion (go).

Corpus Length: the distance from gonion (go) to gnathion (gn).

Symphyseal Height: the distance from gnathion (gn) to infradentale (id).

Symphyseal Thickness: the distance from pogonion (po) to the lingual symphyseal point (syp).

Ramal Width: the distance from the midramal point (mrp) to the midcoronoid process (mcp) point.

Sigmoid Notch Height: the distance

TABLE 2

Number of individuals classified into each of the five Nubian groups (in percent)

\begin{tabular}{lccccc}
\hline $\begin{array}{l}\text { Predicted Group } \\
\text { Classification: }\end{array}$ & A-Group & C-Group & Meroitic & X-Group & Christian \\
\hline Actual Group & & & & & \\
Classification: & 40 & - & 60 & $-\overline{1}$ & $\overline{15}$ \\
A-Group & - & 42 & 26 & 15 & 19 \\
C-Group & 9 & 4 & 46 & 19 \\
Meroitic & 8 & - & 20 & 56 & 14 \\
X-Group & 6 & 6 & 13 & 13 & 59 \\
Christian & & &
\end{tabular}




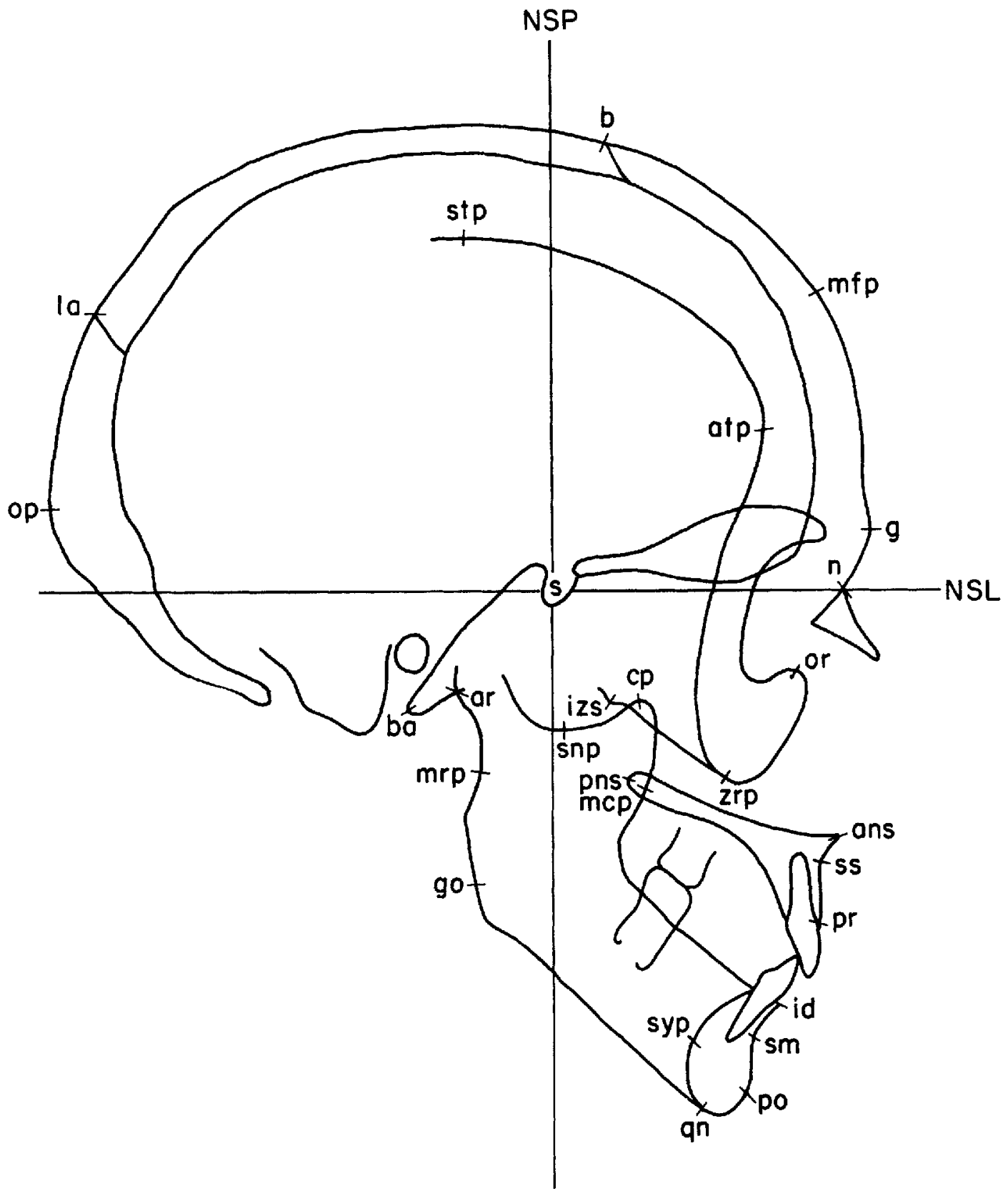

Fig. 2 Diagram indicating the location of the 48 anatomical and derived roentgenographic cephalometric reference points used in this analysis. Each point was recorded as spatial coordinates. Selected sets of reference points were transformed into linear and angular dimensions for statistical analysis. See text for identification of points. 
from gonion (go) to the sigmoid notch point (snp).

Coronoid Process Height: the distance from gonion (go) to the coronoid point (cp).

Mandibular Angle: the angle formed by the mandibular line and a line through articulare (ar) and gonion (go).

Face height and zygomatic process shape

Upper Face Height: the distance from nasion (n) to prosthion (pr).

Morphological Face Height: the distance from nasion (n) to gnathion (gn).

Cheek Height: the distance from orbitale (or) to the zygomaxillary root point (zrp).

Cheek Angle: the angle formed by NSL and a line running through orbitale (or) and the zygomaxillary root point (zrp).

\section{Skeletal-muscular relations}

Anterior Position of Temporalis: the shortest distance from the anterior temporalis point (atp) to nasion-sella perpendicular (NSP).

Temporalis Height: the shortest distance from the superior temporalis point (stp) to NSL.

Anterior Position of Zygomaxillary Root Point: the shortest distance from the zygomaxillary root point (zrp) to NSP.

Masseteric Origin Length: the length of the origin of the masseter muscle, defined as extending from the zygomaxillary root point (zrp) to the inferior zygomaticotemporal suture (izp).

\section{Statistical procedures}

Analysis of the Nubian cranial material included both descriptive, summary statistical procedures and the multivariate technique of multiple discriminant analysis (BMD07M: Dixon, '73). Multiple discriminant analysis provides a means for studying the statistical relationships between populations on the basis of relatively large sets of variable measurements. It identifies and describes major sources of interpopulational variation in terms of discriminant functions, or canonical variates, as well as computing both a measure of the contribu- tion of each variable to a given variate and a measure of the proportion of the total variation explained by each function. A detailed explanation of the mathematical bases and computational procedures involved in the technique of multiple discriminant analysis can be obtained in Morrison ('67), Seal ('64), and Dixon ('73). Two very recent and excellent discussions of the techniques of multiple discriminant analysis and canonical analysis from an anthropological perspective are available in Howells ('72, '73) and Oxnard ('72, '73).

\section{RESULTS}

In a discriminant function between widely distinct populations, only a relatively small amount of overlap among individuals, and thus of misclassification, is likely to occur. In the present study, however, a good deal of overlap between populations is to be expected due to the temporal and hypothesized genetic continuity of the groups. Thus, the degree of discrimination between crania from all five Nubian populations was relatively poor, $44.2 \%$ (table 2). The only interpopulation differences which proved to be significant at the $95 \%$ confidence level occurred between the C-Group and the X-Group samples, the C-Group and the Christian samples, and the X-Group and Christian samples. It is important to note however, that statistical significance does not necessarily indicate widespread biological differences, but only that those interpopulation differences which do occur do so in a consistent manner. Thus, it is critical to examine the patterns of morphological differences between the groups in detail by close inspection of the discriminant functions, or canonical variates.

\section{Discriminant functions}

A maximum of four, $(k-1)$, discriminant functions was possible for consideration in this analysis. Bartlett's Chi-square approximation (Rao, '52: p. 373), however revealed that only three functions were significant at at least the $95 \%$ confidence level. Thus, only the first three functions, 
TABLE 3

Summary table of the three statistically significant $(P<.05)$ discriminant functions, including those variables judged to be significant for the interpretation of each axis

\begin{tabular}{lccc}
\hline \multicolumn{1}{c}{ Function } & I & II & III \\
\hline Eigen value & 2.55 & 0.99 & 0.94 \\
Percent of dispersion & 45.7 & 20.0 & 19.0 \\
Cumulative percentage & 45.7 & 65.7 & 84.7 \\
Measurement & & Scaled vector values \\
\hline Median fossa height & -54.74 & 22.02 & 4.97 \\
Posterior base length & -43.42 & & -4.84 \\
Posterior fossa length & -19.20 & & -4.16 \\
Anterior base length & -11.12 & & \\
Temporalis height & 9.77 & & 18.14 \\
Maxillary length & 8.64 & 5.05 & -5.08 \\
Median base length & -7.86 & 52.14 & -45.25 \\
Upper facial height & 7.63 & -17.10 & 21.50 \\
Upper facial length & -7.54 & & \\
Anterior position of & 6.08 & & \\
$\quad$ zygomaxillary root point & & -19.13 & \\
Masseter origin length & & 10.05 & \\
Cranial height & & -6.17 & \\
Mandibular corpus length & & 5.50 & \\
Frontal chord & & & \\
Anterior position of & & &
\end{tabular}

which combine to account for $84.7 \%$ of the total discrimination (table 3 ), receive further attention.

First function. The first canonical variate was most effective in separating the AGroup and X-Group populations, accounting for nearly half $(45.7 \%)$ of the total dispersion. In addition, with the exception of the Christian population, the groups were ordered according to their appropriate temporal positions along this vector. As seen in figure 3 , the individual discriminant scores for the entire sample overlapped extensively, although the ranges of variation for the A-Group and X-Group were most distinct.

Four measurements of the cranial vault and base were of primary importance in the discrimination of A-Group and $\mathrm{X}$ Group individuals (table 3). These included measurements of median cranial fossa height, posterior cranial fossa height, anterior cranial base length, and posterior cranial base length. Variables describing the upper face were also represented but were of substantially less discriminatory value than the vault and base measurements. All of the vault and base measurements with high scaled vector values exhibited negative signs, indicating that this set of measurements most effectively defined the low (X-Group) end of the first discriminant function. The variables from the upper face were positive, indicating that they were most effective in defining the positive (A-Group) end of the variate.

Second function. The second discriminant function was less than half as effective as the first variate in accounting for interpopulation variation $(20 \%)$. The major distinctions along this axis were drawn between the X-Group and the Christian populations. As with the first function, all of the groups except one, the X-Group in this case, were ordered in the appropriate temporal sequence (fig. 3).

Five variables clustered to account for the Christian end of the second variate (table 3): the length of the origin of masseter, median cranial base length, maxillary length, median cranial fossa height, and the length of the mandibular corpus. Only 

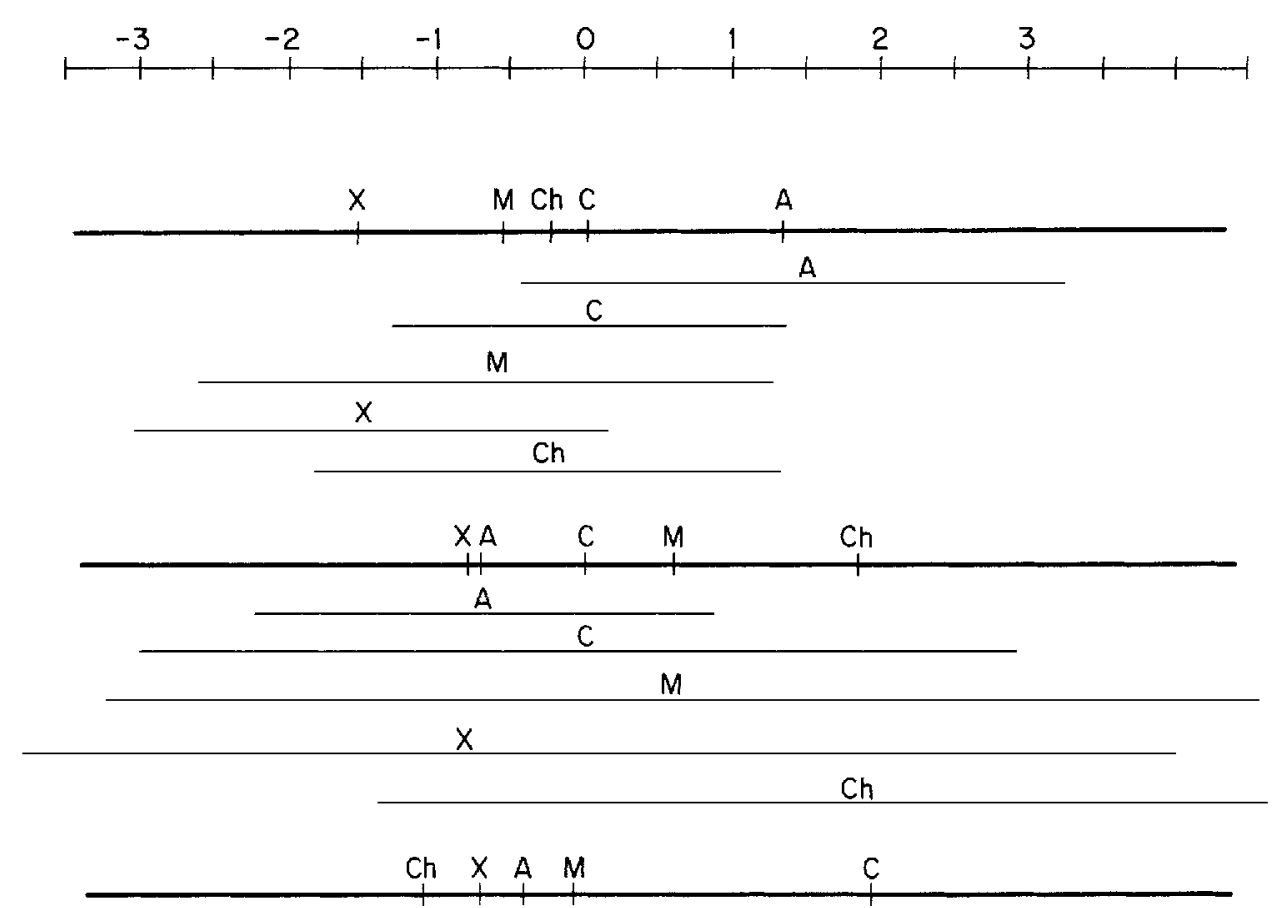

Fig. 3 Plot of the group centroids for the first three discriminant functions (I, II, III). The ranges of variation of the individual discriminant scores to the first standard deviation are indicated for the first two axes.

three variables defined the negative (XGroup) end of the variate: cranial height, upper face length, and the frontal chord.

Third function. The third discriminant function tended to isolate the C-Group population from all of the other groups, but most notably from the Christian population (fig. 3). As with the first two axes, however, the third variate ordered the populations according to their temporal positions with the exception of a single group, the AGroup in this case. This function accounted for the least amount of the total dispersion among the Nubian populations (19\%).

The major differences between crania from the C-Group population and the Christian population, according to this variate, occurred with respect to only three variables: the anterior position of the zygomaxillary root point, masseter origin length, and median cranial base length. The remaining five variables with scaled vector values greater than 4.0 were substantially less important along this axis.
However, it should be noted that the anterior position of temporalis was among them, so that three of the eight highly weighted variables along this axis were directly related to the masticatory musculature.

\section{Interpretation}

The interpretation of the first discriminant function, as well as of subsequent vectors, was facilitated by the graphic display of the means for the craniofacial configurations of each group. The mean spatial coordinates for 48 anatomical and derived points were plotted for each Nubian population. The craniofacial morphologies were then superimposed using a fixed point at sella and the nasion-sella line for purposes of orientation. There are obvious problems related to the use of mean values in an analysis of morphological structure. Implicit within a reliance on mean values is the concept of "type." The present utilization of mean configurations, however, 
DAVID S. CARLSON

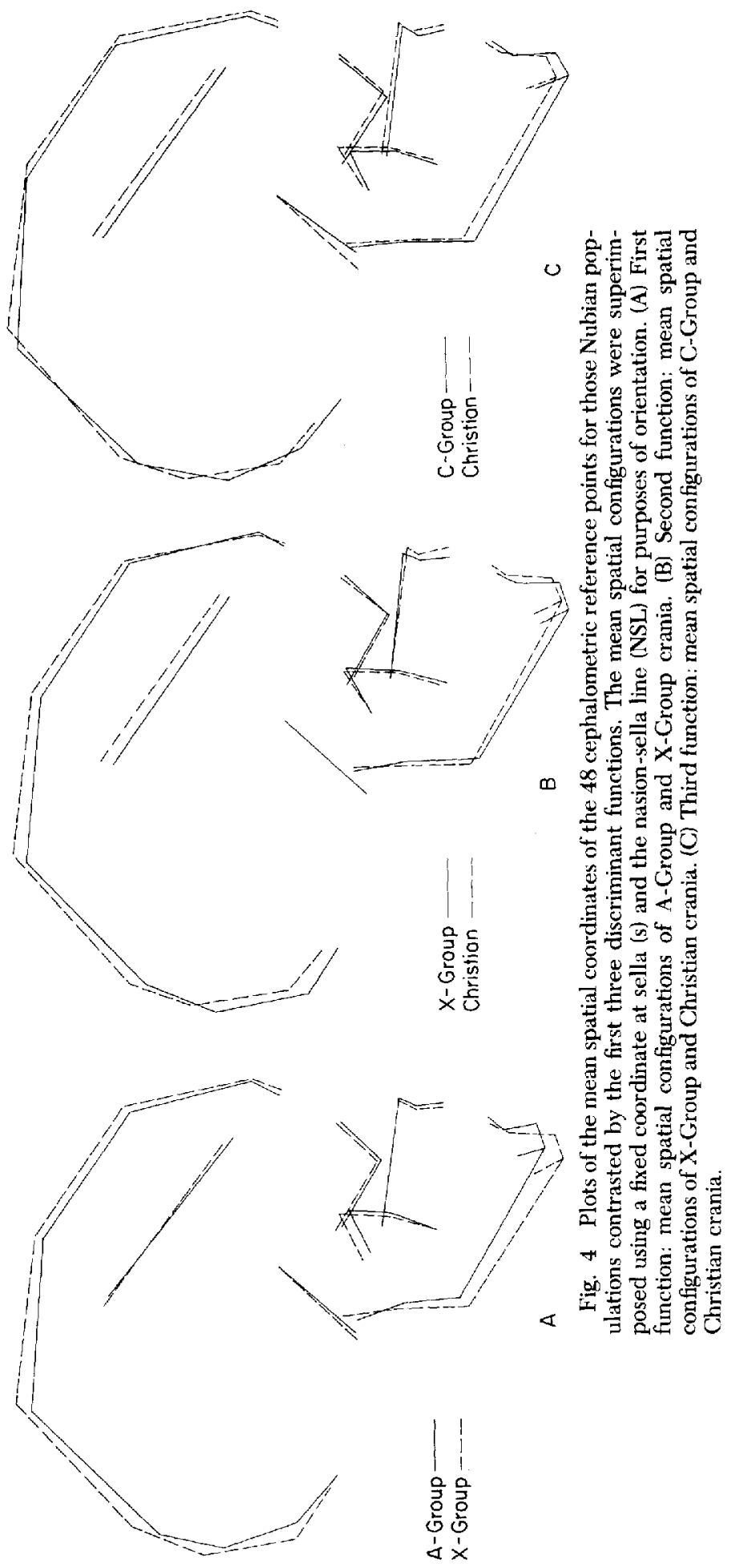


served to enhance the understanding of interpopulation variation among the Nubian populations by providing graphic confirmation to the statistical patterns, as well as providing clues to the total morphological pattern associated with these differences. Furthermore, because the graphic displays of the means for each population were constructed on the basis of mean spatial coordinates for each recorded anatomical point, differences in shape as well as size were maintained within each diagram.

The mean configurations for A-Group and X-Group crania are graphically displayed in figure 4A. Concentrating on those cranial vault measurements with the highest negative scaled vectors scores, some distinct morphological differences between the two populations are apparent. Not only is the A-Group cranial vault generally smaller, but there are differences in the shape of the vault as well. Specifically, the A-Group vault is relatively low and long, with more pronounced glabellar protrusion and a somewhat more pronounced occipital region. The X-Group vault, on the other hand, exhibits a relatively larger anterior cranial fossa, indicated by a slightly more anterior position of the midfrontal point ( $\mathrm{mfp}$ ), and a relatively higher median cranial fossa. The latter is indicated not only by the greater height of bregma (b) from sella (s), but also the fact that lambda (la) occupies an even higher position relative to bregma and lambda in the A-Group. The posterior aspect of the cranial vault for the $\mathrm{X}$-Group, like the rest of the vault, is larger than that of the A-Group in absolute terms. However the shape of the posterior vault in the $\mathrm{X}$ Group is somewhat flatter (more vertical) due to a more superior position of the entire occipital area.

The less substantial positively scaled vector scores for variables relating primarily to the upper face revealed another, though much more subtle, contrast between the A-Group and X-Group populations. The superior temporalis point (stp) is slightly higher for the X-Group in absolute terms, although it is obviously relatively higher in A-Group crania owing to the lower cranial vault in the A-Group. The contrast in the length of the maxilla and in the anterior projection of the zygomaxillary root point (zrp) can be explained in terms of a more anterior projection of the entire zygomaxillary complex in the AGroup population. This accounts, in addition, for the relatively high negative scaled vector score for the length of the upper face. Upper face height, as indicated by the graphic display, is roughly equal for both the A-Group and X-Group. As with the height of temporalis, however, the scaled vector value for upper face height along the first discriminant function accounted for the relative differences between the two groups. Although upper facial height for the A-Group and X-Group was quite similar in absolute terms, the AGroup population had a relatively larger upper face height due to the relatively low height of the cranial vault.

The major differences between the two extreme groups along the first canonical variate can thus be outlined as follows. Distinction between A-Group crania and XGroup crania occurred with respect to the shape of the cranial vault. A-Group crania tended to be relatively lower and longer than X-Group crania. This was manifested by a smaller (more posterior) anterior cranial fossa and a relatively larger posterior cranial fossa for the A-Group. These characteristics, in addition, accounted for the more pronounced glabellar and occipital regions in the A-Group as compared with the X-Group population. The only other difference between the A-Group and $\mathrm{X}$ Group occurred in the zygomaxillary region, which was more anteriorly projected in the A-Group.

Variation among the rest of the Nubian populations can be assumed to follow the same general pattern as that defined by the two extremes along the first variate. The C-Group was most closely associated with the A-Group extreme, while deviating toward the X-Group pattern. Conversely, the Meroitic population was most similar to the X-Group extreme, although it deviated 
toward the A-Group end of the variate. Thus, factors within the cranial vault which contrasted the A- and X-Group populations continued to be modified through the C-Group and Meroitic periods, such that there was a trend from a relatively long, low cranial vault and a slightly more anteriorly projected upper face, to a higher, more anteriorly-superiorly located cranial vault, with a less anteriorly projected upper face. This trend, furthermore, had its beginnings in the A-Group phase, approximately 5,000 years ago, and extended through the X-Group phase, approximately 1,400 years ago.

The mean configurations for the $X$ Group and the Christian populations (fig. $4 \mathrm{~B})$ revealed the general pattern of interpopulation variation described by the second function. The Christian population was characterized by three major morphological features when compared with the $\mathrm{X}$ Group: (1) a smaller origin for the masseter muscle, (2) a larger and slightly more anteriorly placed maxilla, and (3) accompanying a larger maxilla, a longer mandibular corpus. Although variables from the cranial vault and base were relatively less important in the discrimination of the XGroup and Christian crania, the Christian population appeared to emphasize many of the features for the cranial vault and base described for the X-Group in the first function. Specifically, the Christian population had a generally higher and more anteriorly placed cranial vault.

The second discriminant function thus appears to emphasize some of the features related to the shape of the cranial vault initially perceived along the first discriminant axis, while focusing upon variation in the length of the upper face and mandible. The increase in the size of these features took place primarily in their posterior aspects, with the mandibular ramus and the posterior nasal spine occupying a relatively more posterior position.

The third variate was considerably more difficult to interpret than the previous two axes. Some inferences regarding the $\mathrm{C}$ Group-Christian population distinction however, can be drawn from the comparison of their mean configurations (fig. 4C). Three of the eight substantially scored variables defined by the third variate focused upon the muscles of mastication. These included the length of the origin of masseter, the anterior position of the zygomaxillary root point, and the anterior position of temporalis. Some differences in these areas, although subtle, do occur between the C-Group and Christian populations. Specifically, the origin of masseter is more extensive along the zygomatic arch within the C-Group, and the position of the zygomaxillary root point is relatively more anteriorly placed in the C-Group due to the greater forward projection of the frontal bone and maxilla in the Christian population. And, finally, the most anterior aspect of the temporalis muscle is relatively more anteriorly placed in the CGroup owing to the more anteriorly located frontal region in the Christian population. Thus, the third discriminant function differentiated the C-Group from the later X-Group and Christian populations primarily on the basis of the relative position and size of the masticatory musculature.

Two other variables bear consideration from the third function as they relate to the shape of the cranial vault. The general pattern describing cranial vault shape in the first and second functions appears to be re-affirmed by the relatively high scaled vector values for the height of the median cranial fossa. The C-Group contrasts with the Christian population by exhibiting a relatively lower and longer cranial vault. Similar to the A-Group along the first discriminant axis, C-Group crania tend to have a relatively more posteriorly located anterior cranial fossa as well as a more expansive, but lower posterior cranial fossa.

\section{DISCUSSION}

The patterns of morphological variation and statistical ordering of the five Nubian populations revealed by the first three discriminant functions all lend support to the hypothesis that evolutionary change in $\mathrm{Nu}$ - 
bian crania has taken place during the past 5,000 years. The trends indicated by the three statistically independent variates can be summarized most effectively as representing relatively distinct elements of only one broad morphological pattern of variation over time. By far the most important element of the morphological pattern in accounting for variation among the Nubian groups was located along the first discriminant function, and focused on the shape of the cranial vault. Specifically, there was a clear and consistent trend within the Nubian populations to go from a relatively low, elongated cranial vault with a more pronounced glabellar region and occipital area in the earlier groups, to a relatively more globular vault in the latter groups. This statistical observation receives added support from Nielsen's ('70) observation that the Nubian groups have tended to become less dolichocephalic over time. Although all of the populations studied fall within the range for dolichocephaly, the earlier A- and C-Groups are relatively longer and less broad than subsequent groups. Associated also with this change in the shape of the cranial vault over time, according to the second discriminant function, was a less significant tendency for the maxillo-mandibular region to become somewhat more posteriorly located due to an expansion in a posterior dimension. Since the anterior aspect of the maxillomandibular complex remained in approximately the same position, the posterior expansion of the mandibular ramus and of the posterior aspect of the maxilla brought about an overall increase in the length of both the maxilla and of the mandible. The earlier Nubian groups, as well as the $\mathrm{X}$ Group, were also characterized by a slightly more anterior projection of the lower face. However, this projection tended to decrease through the C-Group and Meroitic phases, until the lower face was finally relatively more posteriorly located by the most recent, Christian, phase. The final element of this broad morphological trend is provided by the third canonical axis, which defines temporal variation in the relative size and position of the muscles of mastication. The specific pattern of variation in this regard revealed a tendency for the temporalis muscle to become relatively less anteriorly located as well as for a general reduction in the size of the masseter muscle through time. Thus, the earlier groups had a more anteriorly placed temporalis and larger masseter, while the later groups had a more posteriorly located temporalis and a reduced masseter.

The hypothesis that morphological change in the Nubian skull can be accounted for by the above trends is further supported by a simultaneous consideration of all three canonical axes. As seen in figure 5 , the first two variates maintain the AGroup, the C-Group and the Meroitic populations within a pattern corresponding closely to their relative temporal positions. The third canonical axis orders the later populations, beginning with the C-Group, in their appropriate temporal positions. The location of the A-Group along the third variate can be accounted for by suggesting that the morphological trend defined by the third axis was not complete during A-Group times, but that it culminated during the C-Group and subsequently reversed itself. More specifically, it is suggested that the trend toward a more robust and efficient masticatory musculature was present during the A-Group period, that it reached a peak during CGroup times, and that the muscles of mastication underwent a reduction in the subsequent groups.

A reduction in the robusticity of the muscles of mastication and their associated skeletal elements appears to be a general phenomenon found with behavioral changes associated with change in subsistence patterns. Archeological evidence from Lower Nubia indicates that the AGroup and the early C-Group were not fully adapted to an agricultural economy, and that substantial hunting and gathering did occur (Butzer, '65, '71; Adams, '67). The later Meroitic, X-Group and Christian populations were entirely dependent on an 


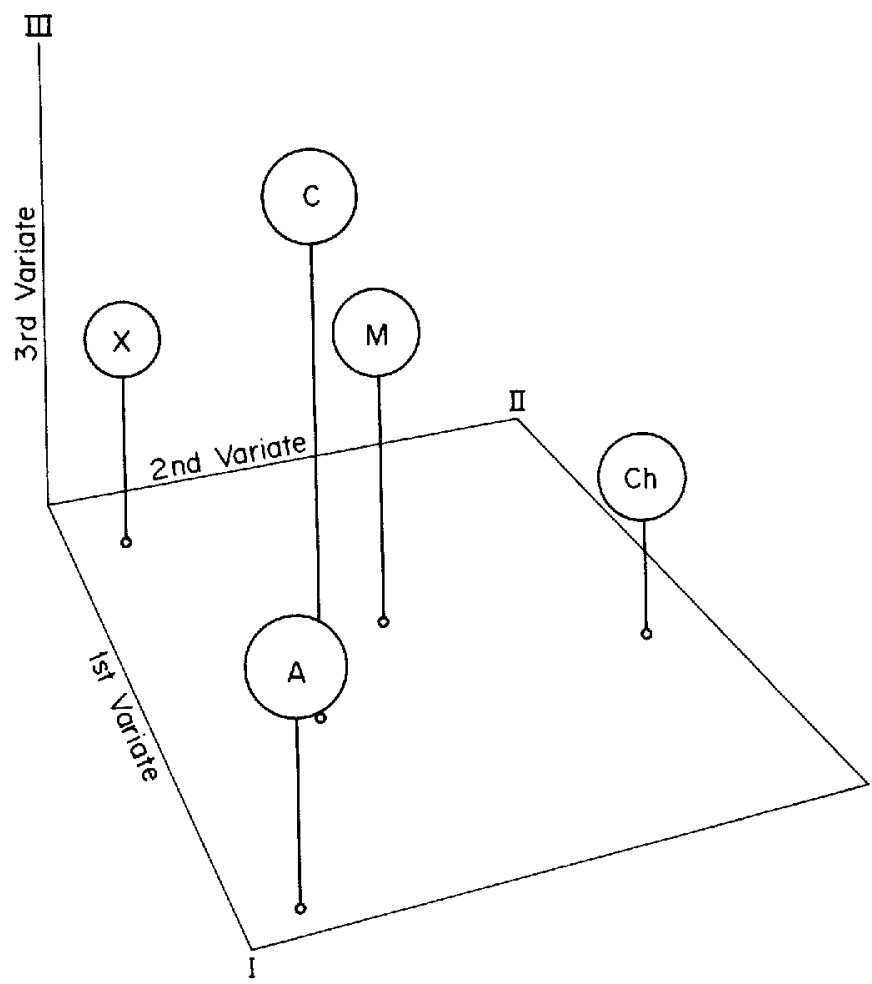

Fig. 5 Three-dimensional model of the distribution of the five Nubian populations along the first three discriminant axes, accounting for $84.7 \%$ of the total variation among the groups.

agricultural subsistence. Thus, the relative robusticity of the muscles of mastication among A-and C-Group peoples probably derives both from their exceptionally robust Mesolithic forebearers (Greene and Armelagos, '72) and their own lifestyle. With increased utilization of softer agriculturally produced foodstuffs there was a concomitant decrease in masticatory robusticity through time.

Recent work by Hylander ('72) adds to an understanding of the relationship between masticatory stress and skeletal morphology. Hylander ('72) noted that a relatively more anterior position of temporalis and of the anterior origin of masseter (zygomaxillary root point) increases the biomechanical efficiency with which the muscles of mastication function. Thus, the relatively more anterior origins of temporalis and masseter in the $\mathrm{A}$ - and $\mathrm{C}$ Groups adds further support to the hy- pothesis that individuals in these groups required greater functional efficiency in mastication, and that the subsequent morphological changes between these and later groups are due, in part, to altered masticatory function.

The nature of craniofacial change in Nubia between the A-Group and Christian periods can be further evaluated through inspection of the mean spatial configurations from each culture group juxtaposed with its temporal predecessor and successor. As can be seen in figure $6 \mathrm{~A}$, the cranial vault in the transition from the AGroup to the C-Group became relatively higher and somewhat shorter. The maxillomandibular complex became more posteriorly located, due primarily to an increase in the size of the maxilla and mandible at their posterior aspects. And finally, the length of the origin of the masseter muscle appeared to have increased slightly in the 

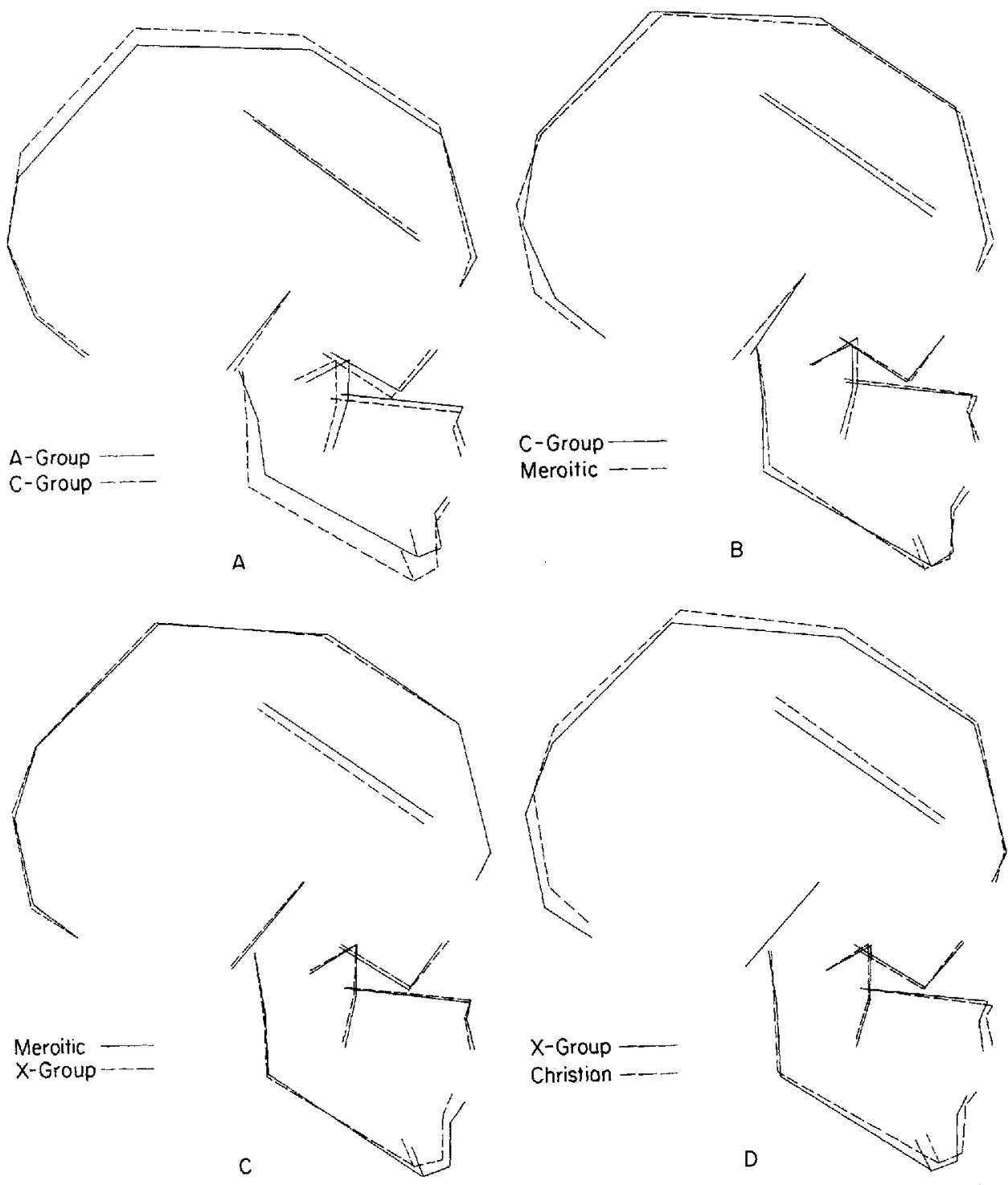

Fig. 6 Plots of the mean spatial configurations of crania from five Nubian populations. Each is superimposed on its predecessor in order to indicate the morphological trends present throughout the approximately 5,000 year time span in Lower Nubia. The orientation is the same as in Figure 4. (A) Superimposition of mean spatial coordinates for A-Group and C-Group crania. (B) Superimposition of mean spatial co-ordinates for C-Group and Meroitic crania. (C) Superimposition of mean spatial coordinates for Meroitic and X-Group crania. (D) Superimposition of mean spatial coordinates for XGroup and Christian crania.

C-Group, while the position of temporalis became slightly more anteriorly situated relative to the frontal region and to the $\mathrm{zy}$ gomaxillary root point.

The C-Group and Meroitic populations were very similar with respect to all three discriminant functions. This was reflected in the comparison of their graphic configurations (fig. $6 \mathrm{~B}$ ), as well as in their relative group centroid locations along the first three variates. Although some minor differences between these two groups can be 
perceived on the basis of their mean values, the group centroids indicated a continuation of the trend away from a low, elongated vault as well as a continuation of the trend for a greater posterior expansion of the lower face and an alteration in the size and position of the muscles of mastication.

The transition from the Meroitic to XGroup populations was marked by the close similarities of their cranial vault shape. The morphology of the lower face in both groups was relatively distinct, however, and indicated a tendency for a substantial relocation of the maxillo-mandibular complex to a more posterior position (fig. 6C). In addition, some difference with respect to the relative size and position of the temporalis muscle was indicated for these two groups by their locations along the third canonical variate. The Meroitic population appears to have a somewhat larger and more anteriorly located temporalis. No substantial differences in the size of the origin of the masseter muscle are apparent from their mean configurations.

The X-Group and Christian populations (fig. 6D) were characterized by their close similarities along the third canonical variate, and their relatively sharp distinctions with respect to the first and especially the second axes. It is evident that the trend toward a shorter and higher cranial vault, as suggested along the first discriminant axis, continued into the Christian population. Similarly, the lower face tended to continue to become somewhat more posteriorly located due to a posterior translation of the posterior border of the ascending ramus of the mandible of the posterior nasal spine. However, associated with this trend in the Christian population was an increase in the anterior projection of the maxilla and mandibular corpus.

\section{CONCLUSION}

Although several studies of skeletal remains of the indigenous populations of Lower Nubia have emphasized the likelihood of direct biological continuity be- tween them, none of these studies have closely examined the patterns of interpopulation variation over time. Most have simply provided an estimate of the probability that each cultural horizon was biologically distinct from the others. The present analysis of the patterns of craniometric variation between temporally successive populations of Lower Nubia further supports the hypothesis that the groups are biologically continuous by focusing on the nature of morphological change over approximately 5,000 years. The added advantage of this approach is that questions concerning the factors responsible for craniofacial change through time can be addressed more easily.

Variation in the Nubian skull over the past 5,000 years has been maintained within one broad morphological trend which is composed of no more than three relatively independent morphological patterns. The trend from a relatively elongated and low cranial vault with a more prognathic lower face, to a shorter and higher (more globular) vault and a less prognathic lower face indicate a general and progressive "rotation" of the entire craniofacial complex through time. The face of the indigenous Nubians became more inferior-posteriorly located with respect to the cranial vault. Additionally, there was a general reduction in the robusticity of the muscles of mastication and a slight positional change in the origins of these muscles which could indicate changing demands on masticatory function through time.

The precise relationship between the form and function of the Nubian craniofacial complex cannot be ascertained directly from this study. Elucidation of this problem is the subject of present research on the form and function of the masticatory complex both in early Nubians and under experimental conditions using laboratory animals. However, the present analysis does provide strong indirect evidence equating change in the Nubian craniofacial skeleton and functional-behavioral alteration, which provides a framework for posing meaningful questions about the rela- 
tionship of form and function in the craniofacial complex. For example, reduction of the prominence of the glabellar and occipital regions, which have been related directly (Wolpoff, '75) to masticatory stress, is also the result of the spatial change between the cranial vault and face seen in the Nubians. Furthermore, the reduction in masticatory robusticity and concomitant changes in craniofacial morphology between approximately 3,400 B.C. and 1,500 A.D. is a logical continuation of changing adaptations from the Nubian Mesolithic (Greene and Armelagos, '72). The present analysis thus draws more explicit attention to two major points: (1) that the craniometric differences between the indigenous populations of Lower Nubia over the past 5,000 to 12,000 years can be perceived most effectively within an evolutionary, processual framework; and (2) that change in craniofacial morphology among the Nubians is most likely due to progressive changes in the functional demands placed on the masticatory complex. This latter conclusion now stands as a working hypothesis in need of further analysis.

\section{ACKNOWLEDGMENTS}

Much of the work done on this project was undertaken while a George C. Marshall Fellow, and I would like to thank the Danish-American Foundation for their support.

I would also like to express my appreciation to Doctors Ole Vagn Nielsen, Arne Björk, Baslev Jørgensen, and Brūno Froelich for their cooperation and assistance during my research at the University of Copenhagen. Thanks also to Ms. Carole Bride for her assistance in preparing this manuscript.

\section{LITERATURE CITED}

Adams, W. Y. 1966 The Nubian campaign; Retrospect and prospect. Mélanges Offerts a Kazimierz Michalowski. Warsaw, pp. 13-20. 1967 Continuity and change in Nubian culture history. Sudan Notes and Records, 48: 1-32. 1968 Invasion, diffusion, evolution? Antiquity, 42: 194-215.
1970 A re-appraisal of Nubian culture history. Orientalia, 39: 269-277.

Arkell, A. J. 1961 A History of the Sudan. Atholone Press, London.

Batrawi, A. M. 1935 Report on the human remains. Mission Archéologique de Nubie 1929-1934. Government Press, Cairo.

1946 The racial history of Egypt and Nubia, Part II. J. Roy. Anth. Inst. 76: 132-156.

Baumgartel, E. J. 1965 Predynastic Egypt. In: The Cambridge Ancient History. Cambridge Univ. Press, Cambridge.

Berry, A. C., and R. J. Berry 1972 Origins and relationships of the ancient Egyptians. Based on a study of non-metrical variations in the skull. J. Hum. Evol., 1: 199-208.

Berry, A. D., R. J. Berry and P. J. Ucko 1967 Genetical change in ancient Egypt. Man 2: 551-568.

Burnor, D. R, and J. E. Harris 1968 Racial continuity in Lower Nubia: 12,000 to the present. Proceedings of the Indiana Academy of Science 1967, 77: 113-121.

Butzer, K. W. 1965 The Cambridge Ancient History. Vol. 1, chapt. 2. Cambridge Univ. Press, Cambridge.

1971 Environment and Archeology: An Ecological Approach to Prehistory. Second ed. Aldine, Chicago.

Collett, M. A. $1933 \quad$ A study of Twelfth and Thirteenth Dynasty skulls from Kerma (Nubia). Biometrika, 25: 254-285.

Crichton, J. M. 1966 A multiple discriminant analysis of Egyptian and African Negro crania. Papers of the Peabody Mus. of Arch. and Ethnol. Harvard Univ, 57(1): 43-67.

Derry, D. E. 1909 Anatomical report (B). Bulletin of the Archeological Society of Nubia, III: 29-52.

Dixon, W. J., ed. 1973 BMD. Biomedical Computer Programs. Univ. of California Press, Berkeley.

Greene, D. L. 1966 Dentition and the biological relationships of some Meroitic, X-Group, and Christian populations from Wadi Halfa, Sudan. Kush, 14: 285-288.

1972 Dental anthropology of early Egypt and Nubia. J. Hum. Evol., 1: 315-324.

Greene, D. L., and G. J. Armelagos 1972 The Wadi Halfa Mesolithic Population. Research Report No. 11. Department of Anthropology, University of Massachusetts, Amherst.

Holthoer, R., 1. Ojamaa-Koskimen, T. SäveSöderbergh 1973 Pharonic New Kingdom Sites. Scandinavian Joint Expedition to Sudanese Nubia, Vol. 5, Scandinavian University Books. Munksgaard, Copenhagen.

Howells, W. W. 1972 Analysis of patterns of variation in crania of recent man. In: The $\mathbf{F u n c t i o n a l ~ a n d ~}$ Evolutionary Biology of Primates. R. Tuttle, ed. Aldine, Chicago. pp. 123-151.

- 1973 Cranial Variation in Man. Peabody Museum of Archeology and Ethnology, Harvard University. Vol. 67.

Hylander, W. L. 1972 The Adaptive Significance 
of Eskimo Cranio-facial Morphology. Ph.D. Dissertation, Univ, of Chicago.

Junker, H. 1926 The first appearance of the Negroes in history. J. Egypt. Arch., 7: 121-132.

Krogman, W. M., and V. Sassouni 1957 A Syllabus in Roentgenographic Cephalometry. Philadelphia Center for Research in Child Growth. Philadelphia.

Lasker, G. W. 1972 The potential relevance of studies of ancient Egyptian populations for the microevolutionary study of modern populations. J. Hum. Evol., 1: 137-139.

MacGaffey, W. 1966 Concepts of race in the historiography of Northeast Africa. J. Afr. Hist., 7: 1-17.

Masali, M. 1972 Body size and proportions as revealed by bone measurements and their meaning in environmental adaptation. J. Hum. Evol., 1: 187-197.

Møllerup, O., and E. Oldenburg 1973 Late Nubia Cemetaries. Scandinavian Joint Expedition to Sudanese Nubia. Vol. 6. Scandinavian University Books. Munksgaard, Copenhagen.

Morant, G. M. 1925 A study of Egyptian craniology from prehistory to Roman times. Biometrika, 17: 1-52.

1935 A study of pre-dynastic Egyptian skulls from Badari based on measurements taken by Miss B. N. Stoessiger and Prof. D. E. Derry. Biometrika, 27: 293-308.

Morrison, D. F. 1967 Multivariate Statistical Methods. McGraw-Hill, New York.

Mukherjee, R., C. R. Rao and J. C. Trevor 1955 The Ancient Inhabitants of Jebel Moya (Sudan). University Press, Cambridge.

Nielsen, O. Vagn 1970 The Nubian Skeleton Through 4,000 Years. Thesis, Andelsbogtrykkeriet i Odense, Denmark.

Nordstrom, H. A., and R. Haland 1972 Neolithic and A-Group Sites. Scandinavian Joint Expedition to Sudanese Nubia, Vol. 3. Scandinavian University Books. Munksgaard, Copenhagen.

Nordström, H. A., and I. Ojama-Koskinen 1973 C-Group, Pangrave and Kerma Sites. Scandinavian Joint Expedition to Sudanese Nubia. Scandinavian University Books. Munksgaard, Copenhagen.

Oxnard, C. E. 1972 Functional morphology of pri- mates: Some mathematical and physical methods. In: The Functional and Evolutionary Biology of Primates, R. Tuttle, ed. Aldine, Chicago, pp. 305-336.

1973 Form and Pattern in Human Evolution. Univ. of Chicago Press, Chicago.

Reisner, G. A. 1909 The Archeological Survey of Nubia. Bulletin No. 3. Gov't. Press, Cairo.

Seal, H. 1964 Multivariate Statistical Analysis for Biologists. Methuen and Co., London.

Säve-Söderbergh, T. 1962 Preliminary report of the Scandinavian Joint Expedition. Kush, 10: 76-105.

1963 Preliminary report of the Scandinavian Joint Expedition. Kush, 11: 47-69.

1964 Preliminary report of the Scandinavian Joint Expedition. Kush, 12: 25-29.

Stoessiger, B. N. 1927 A study of the Badarian crania recently excavated by the British School of Archeology in Egypt. Biometrika, 19: 110-150.

Strouhal, E. 1971 A contribution to the anthropology of the Nubian X-Group. Anthropological Congress Dedicated to Ales Hrdlicka, 1969, Praha, Humpolec: 541-547.

Van Gerven, D. P., D. S. Carlson and G. J. Armelayos 1973 Racial history and bio-cultural adaptation of Nubian archeological populations. J. Afr. Hist., 14: 555-564

de Villiers, H. 1968 The Skull of the South African Negro. Johannesburg.

Walker, G. F. 1972 A new approach to the analysis of craniofacial morphology and growth. Am. J. Orthod., 61: 221-232.

Walker, G. F., and C. J. Kowalski 1971 A two-dimensional model for the quantification, description analysis, prediction and simulation of craniofacial growth. Growth, 35: 191-211.

Wiercinski, A. 1967 Analiza struktury rasowej ludnosci Egiptn w epoce przeddynastyczej. Materialy i pace antropologiczne, No. 56 Panstwowe wydawnnictwo naukowe, Wroclaw.

Wolpoff, M. H. 1975 Some aspects of human mandibular evolution. In: Determinants of Mandibular Form and Growth. J. A. McNamara, ed. Center for Human Growth and Development, Craniofacial Growth Series Monograph No. 4, Ann Arbor. pp. 1-64. 